ARTICLE

https://doi.org/10.1038/s41467-019-11466-5

\title{
Evaporation-induced sintering of liquid metal droplets with biological nanofibrils for flexible conductivity and responsive actuation
}

\author{
Xiankai $\mathrm{Li}^{1,2}$, Mingjie Li(i) ${ }^{1}$, Jie $\mathrm{Xu}^{1,2}$, Jun You' ${ }^{1}$ Zhiqin Yang ${ }^{3} \&$ Chaoxu $\mathrm{Li}^{1,2}$
}

Liquid metal (LM) droplets show the superiority in coalescing into integral liquid conductors applicable in flexible and deformable electronics. However, the large surface tension, oxide shells and poor compatibility with most other materials may prevent spontaneous coalescence of LM droplets and/or hybridisation into composites, unless external interventions (e.g., shear and laser) are applied. Here, we show that biological nanofibrils (NFs; including cellulose, silk fibroin and amyloid) enable evaporation-induced sintering of LM droplets under ambient conditions into conductive coating on diverse substrates and free-standing films. The resultants possess an insulating NFs-rich layer and a conductive LM-rich layer, offering flexibility, high reflectivity, stretchable conductivity, electromagnetic shielding, degradability and rapid actuating behaviours. Thus this sintering approach not only extends fundamental knowledge about sintering LM droplets, but also starts a new scenario of producing flexible coating and free-standing composites with flexibility, conductivity, sustainability and degradability, and applicable in microcircuits, wearable electronics and soft robotics.

\footnotetext{
${ }^{1}$ Group of Biomimetic Smart Materials Qingdao Institute of Bioenergy and Bioprocess Technology Chinese Academy of Sciences, Qingdao 266101 , China. ${ }^{2}$ Center of Material and Optoelectronics Engineering University of Chinese Academy of Sciences, Beijing 100049, China. ${ }^{3}$ School of Materials Science and Engineering Harbin Institute of Technology, Harbin 150001, China. Correspondence and requests for materials should be addressed to M.L. (email: limj@gibebt.ac.cn) or to C.L. (email: Licx@qibebt.ac.cn)
} 
$\mathrm{N}$ anomaterial agglomeration and coalescence are of great importance for the formation of interconnecting conductive networks of flexible electronics applicable in soft robotics, stretchable, wearable, implantable and biomedicine devices $^{1,2}$. Many conductive $0-1$ dimensional (0-1D) nanomaterials (e.g., nanotubes, nanoparticles and nanowires of metals ${ }^{3}$, carbon $^{4}$ and conjugated polymers ${ }^{5,6}$ ) have been endeavoured either as the colloidal inks for deposition, writing and printing, or as the fillers in organic composites ${ }^{7}$. When depositing and printing solid conductive colloids, capillary forces induced by solvent evaporation could assist to organise them into closelypacking layers ${ }^{8}$. When incorporating solid nanomaterials into organic matrix, large filler compositions were normally required to exceed the percolation threshold and form the interconnecting networks of charge transport ${ }^{3,9,10}$, e.g., 16.2 vol\% for Au nanoparticles in polyurethane ${ }^{9}$ and $10 \mathrm{wt} \%$ for carbon black in polyester ${ }^{10}$. In most cases, agglomeration and coalescence of rigid solid nanomaterials may show contact resistance of charge transport at their physical junctions. The encapsulated layers of solid nanomaterials, which were essential for colloidal stability and compatibility with organic matrix, may also hinder their direct contact and hereby charge transport.

In contrast to rigid solid nanomaterials, droplets of liquid metal (LM, e.g., EGaIn with $75 \%$ gallium and $25 \%$ indium $^{8}$ ) show the superiority in sintering into integral liquid conductors which could withstand physical deformation as diverse as bending, twisting, stretching and compression ${ }^{11,12}$. EGaIn droplets could be stabilised and incorporated into the printable inks and organic matrix, under the shell protection of the oxide layer (spontaneously forming and with the thickness of $0.5-3 \mathrm{~nm}^{12}$ ), ligand coordination and microgels ${ }^{11,13}$. Thus great potential of LM has been promised in flexible electronics because of its fluidity, metallic conductivity, negligible vapour pressure and low toxicity ${ }^{12,14,15}$. However, when applying in deposition, writing, printing ${ }^{16}$, injecting into microchannels and embedding into organic matrix, EGaIn droplets were normally hindered from closely packing and spontaneously sintering due to the combination of high surface tension $\left(624 \mathrm{mN} \mathrm{m}^{-1}\right)$ of EGaIn, protection shells and incompatibility with other materials. In particular, structural cracks frequently emerged within the layers of LM droplets due to internal stress produced by unsymmetrical capillary forces ${ }^{8,11}$. External interventions were usually required to rupture their encapsulating shells and release fluidic LM for droplet sintering, such as mechanical sintering 8,17 and laser sintering ${ }^{18,19}$. But it still remains far from being fully understood how various factors control the sintering processes of LM droplets for the formation of conductive layers on diverse substrates and interconnecting networks within organic matrix.

Recently, it was suggested that capillary forces could produce a local pressure at nanoscale up to $10^{1} \sim 10^{3} \mathrm{MPa}$ at the junctions of $\mathrm{Ag}$ nanowires (with the diameter of $50-90 \mathrm{~nm}$ ), being sufficient for cold welding of the nanowires ${ }^{20,21}$. Herein we show that evaporation at the ambient condition (room temperature of $\sim 20^{\circ} \mathrm{C}$, ordinary pressure of $\sim 0.1 \mathrm{MPa}$ and relative humidity (RH) of $\sim 40 \%$ ) can sinter colloidal suspensions of EGaIn droplets in the presence of biological nanofibrils (NFs, with the diameter of $\sim 5-10 \mathrm{~nm}$ ) as low as $0.05 \mathrm{wt} \%$ of cellulose, silk fibroin and amyloid, and hereafter produce conductive layers on diverse substrates and free-stranding composites with multiple functionalities, such as optical reflectivity, flexible conductivity (up to $8.9 \times 10^{5} \mathrm{~S} \mathrm{~m}^{-1}$ ) and rapid responsive actuation. In contrast to conventional mechanical sintering solely on rigid substrates and laser sintering on heat-resistant substrates ${ }^{8,18}$, this spontaneous sintering method can serve for conductive patterns, circuits and electromagnetic shield on both rigid and soft substrates (e.g., elastic, inorganic and biological). When sintering on elastic substrates, the EGaIn coating endures a tensile stain up to $200 \%$ without showing clear conductivity decay. The consolidating product may also be free-stranding with the thickness up to tens of micrometres. Consisting of a bottom EGaIn-rich layer and a top NFs-rich layer, the resultant Janus film can respond to low voltage $(<3 \mathrm{~V})$, photo and humidity, and show actuating behaviour with great bending speed (e.g., $120^{\circ} \mathrm{s}^{-1}$ ) and repeatability, being comparable with or superior to most of soft actuators in the literature.

\section{Results}

Design strategies. Biological NFs with the diameter of $<10 \mathrm{~nm}$ are widely distributed in biomasses (Fig. 1a and Supplementary Fig. 1,2), and function in living organisms for mechanical support (e.g., in crab shell), protection (e.g., in silk cocoon), adhesion (e.g., in biological membrane) and pathogenesis (e.g., related to Alzheimer's diseases) ${ }^{22}$. Technologically, silk NFs and amyloid NFs could be produced through supramolecular self-assembly (Supplementary Methods and Supplementary Fig. 1) 23,24. Cellulose NFs (CNFs), with the average diameter of $\sim 6 \mathrm{~nm}$ and aspect ratio of $>10^{2}$ (Fig. 1a and Supplementary Fig. 2), could be exfoliated from hardwood through a 2,2,6,6-tetramethylpiperidyl-1oxyl (TEMPO)-mediated oxidisation ${ }^{25}$. With the advantages of low cost and sustainability, these NFs have seen promising applications in biomedicine, catalysis, reinforcing fillers, optoelectronics and energy-harvest ${ }^{25}$.

Among these NFs, the synthesised CNFs typically had plentiful carboxyl groups (with the content up to $1.4 \mathrm{mmol} \mathrm{g}^{-1}$ ) and hereby negatively charged surfaces (e.g., $\zeta$-potential $\sim-50 \mathrm{mV}$ at pH 7 shown in Supplementary Fig. 2, 3), being capable of forming a stable aqueous suspension (e.g., with the concentration $\phi_{\mathrm{CNF}}=$ $0.5 \mathrm{wt} \%)$ at the nematic liquid-crystalline state. EGaIn, acting as a typical eutectic alloy (with a melting point $\sim 15.8^{\circ} \mathrm{C}$ ), remains fluidic at room temperature with a large surface tension $(624 \mathrm{mN}$ $\left.\mathrm{m}^{-1}\right)$ and high conductivity $\left(3.4 \times 10^{6} \mathrm{~S} \mathrm{~m}^{-1}\right)$. When sonicating ( $300 \mathrm{~W}$ and $20 \mathrm{kHz}$ ) EGaIn in the suspension of CNFs, an opaque grey slurry was produced with the formation of EGaIn droplets (Fig. 1b). By optimising the sonication time (e.g., $60 \mathrm{~min}$ in Supplementary Fig. 4) and CNFs concentration (e.g., $\phi_{\mathrm{CNF}}=0.2$ $w t \%$ in Supplementary Fig. 5), the average droplet size could decrease down below $100 \mathrm{~nm}$ (Fig. 1c). The average droplet diameter of $50 \mathrm{~nm}$ was possibly achieved via further centrifugation (5000 rpm) for size grading (Supplementary Fig. 6).

To be noted, without the presence of CNFs, aqueous suspensions of EGaIn droplets were also produced with the size of $>400 \mathrm{~nm}$ (Supplementary Fig. 5). It was reported that spontaneous oxidation assisted to encapsulate EGaIn droplets within a thin oxide shell ${ }^{15}$. In the presence of CNFs, EGaIn droplets were further stabilised by binding a certain amount of CNFs on their surface via crosslinking and coordination of carboxyl groups with $\mathrm{Ga}$ oxydate like $\mathrm{Ga}^{3+}$ (Fig. 1c and Supplementary Fig. 7) ${ }^{11}$. Thus the resultant suspension had the smaller EGaIn droplets and was capable of maintaining stable up to days at $\mathrm{pH} \sim 7$ under $\mathrm{N}_{2}$ protection, with negligible precipitation and chemical oxidation (Supplementary Fig. 8, 9).

When casting the suspension of EGaIn droplets under the ambient condition, a free-standing bilayer film was obtained with the micrometric thickness. The grey top layer consisted mainly of CNFs, while the bottom layer consisted mainly of sintered EGaIn and showed smooth mirror-like surfaces (Fig. 1d, e, Supplementary Fig. 10 and Supplementary Movie 1). The thickness ratio of these two layers could be tuned by controlling the concentration ratio of CNFs and EGaIn in the suspension. Besides the freestanding films, EGaIn droplets together with CNFs could also deposit on various substrates through mask-printing, 

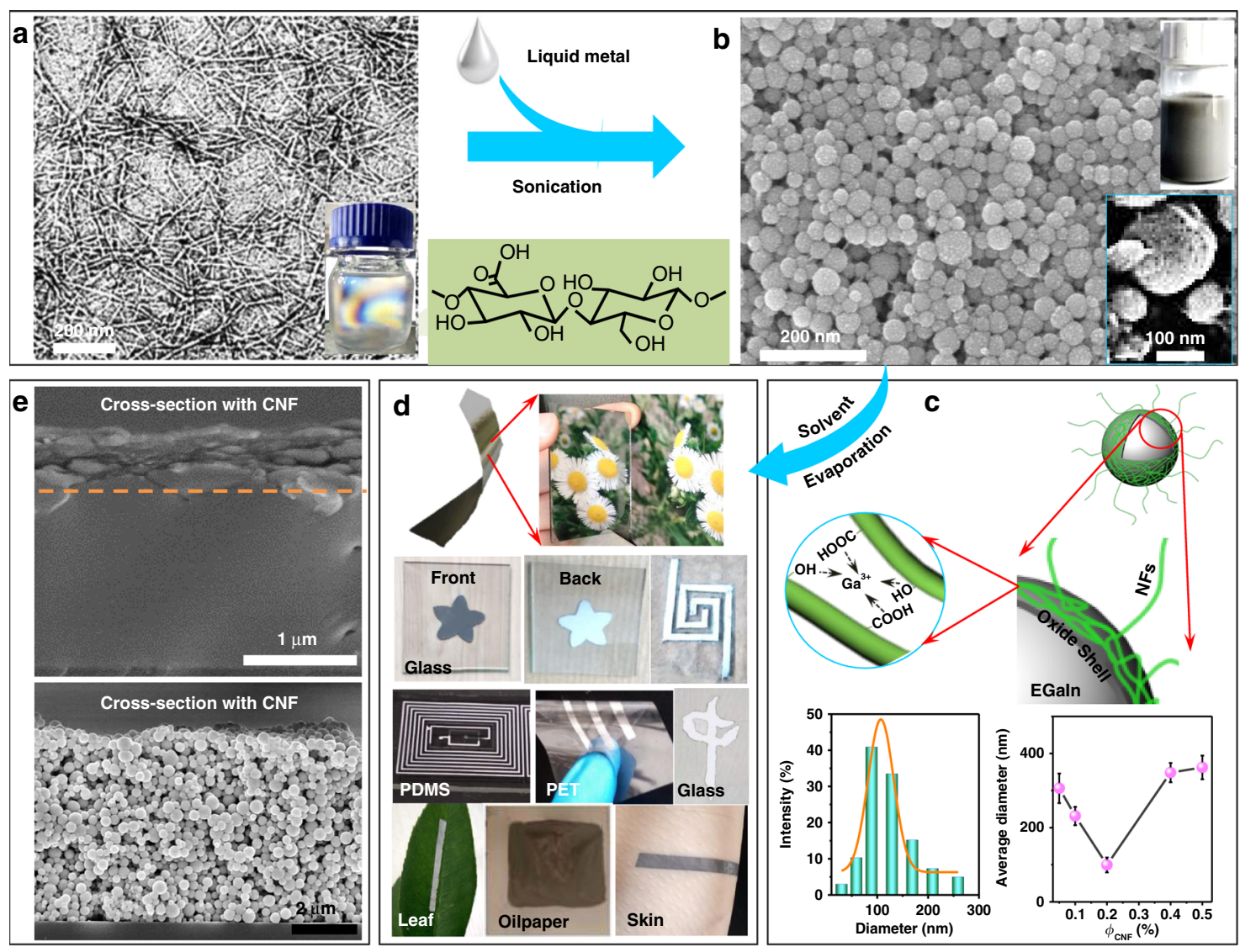

Fig. 1 Evaporation-induced sintering of EGaln droplets with biological NFs for free-standing films and coatings. Evaporation-induced sintering of EGaln droplets with biological NFs. a Typical transmission electron microscopy (TEM) image of CNFs. The inset gives optical image of nematic CNFs suspension. b Typical scanning electron microscopy (SEM) image of EGaln droplets produced by sonicating bulk EGaln in CNFs suspension. The top-right inset gives corresponding optical image. Sonication: $60 \mathrm{~min}$; Concentration $\left(\phi_{\mathrm{CNF}}\right): 0.2 \mathrm{wt} \%$. c Schematic illustration of EGaln droplet encapsulated in oxide shell and with CNFs attached on surface via interactions with $\mathrm{Ga}^{3+}$. Diameter histogram of EGaln droplets in $\mathbf{b}$ (Bottom left) and effect of $\phi_{\mathrm{CNF}}$ on average EGaln diameter after $60 \mathrm{~min}$ sonication (Bottom right) were given as the inset. $\mathbf{d}$ Evaporation-induced sintering into free-standing films (Top) with mirror-like bottom surface and grey top surface, and coatings (Bottom) on different substrates through mask depositing, hand-writing or drop-casting. e Crosssectional SEM images of coating layers of EGaln droplets with (Top) and without CNFs (Bottom)

channel-depositing, hand-writing and drop-casting (Fig. 1d, Supplementary Fig. 11). A bilayer coating was produced (Fig. 1d and Supplementary Fig. 12), either on the rigid substrates (e.g., glass and mica) or on the soft substrates (e.g., polyethylene terephthalate (PET), polypropylene (PP), styrene-ethylenebutylene-styrene (SEBS), polydimethylsiloxane (PDMS), leaf and skin). In contrast, without biological NFs, no free-stranding film or coating was produced either via casting or via filtration (Supplementary Fig. 13). And EGaIn droplets were deposited only randomly on the substrate without showing conspicuous coalescence and sintering (Fig. 1e, Supplementary Fig. 13). Similar results were also obtained by replacing CNFs with silk NFs or amyloid NFs (Supplementary Fig. 14).

Flexible conductivity \& degradability. Both the free-standing films and coating on different substrates shared a common bilayer structure (Fig. 1e), suggesting the presence of a precipitation separation process during the evaporation (Supplementary Fig. 15, 16). Due to the high density $\left(6.28 \mathrm{~g} \mathrm{~mL}^{-1}\right)^{14}$, EGaIn droplets precipitated first and sintered into the bright layer with an electric conductivity (Supplementary Fig. 14). CNFs had relatively high colloidal stability and deposited on the EGaIn layer as the insulating layer. Inevitably, CNFs might also exist within the conductive layer because of their presence on and between the EGaIn droplets during evaporation-induced sintering
(Supplementary Fig. 17a). EGaIn droplets, when having the smaller size and attaching more CNFs, might also exist within the insulating layer because of their higher colloidal stability (Supplementary Fig. 17, 18).

The presence of CNFs not only assisted to sinter the EGaIn droplets via evaporation, but also served as the structural support for comparable mechanical properties of the free-standing Janus films (e.g., an elastic modulus up to $4.75 \mathrm{GPa}$ in Supplementary Fig. 19a, b). The remaining CNFs within the EGaIn-rich layer also enabled its endurance to mild rub (see the inset of Fig. 2a), in contrast to weak surfaces when lacking CNFs (Supplementary Fig. 19c). The bottom bright layer reflected light in a way akin to bulk LM with a reflection $\geq 80 \%$ within the visible-light region $(400-720 \mathrm{~nm})$, whilst the top CNFs layer displayed high light absorption and thus a low reflection $\leq 20 \%$ (Fig. 2a and Supplementary Fig. 20).

When depositing on elastic substrates (e.g., SEBS), the EGaIn coating could survive hundreds of cycles of bending and twisting, without showing sensible conductivity decay (Fig. 2b and Supplementary Fig. 21). More surprisingly, this conductive layer adhered closely to the substrate during cyclic stretching, and maintained highly conductive with the strain up to $200 \%$ (Fig. $2 \mathrm{c}$ and Supplementary Fig. 22), in spite that the top CNFs-rich layer peeled off from the coating. In sharp contrast, the EGaIn coating without CNFs formed isolated granular morphologies after 

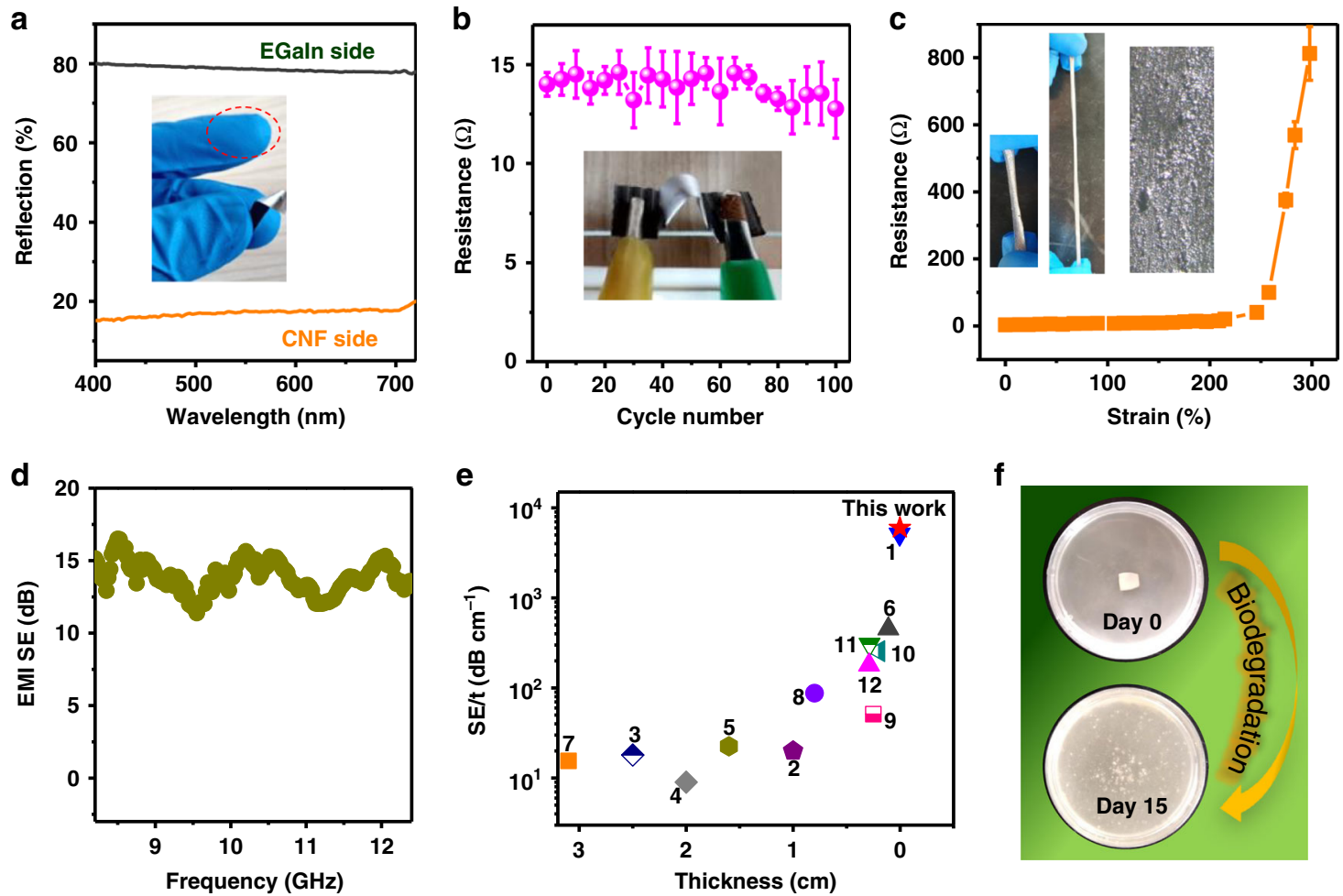

Fig. 2 Physical characterisation of Janus EGaln film and coating. a Optical reflection of CNFs-rich and EGaln-rich sides. The inset gives endurance of EGaln side to mild rub. b Cyclic bending endurance evaluated by electric resistance. EGaln/CNFs layer thickness: $9 / 10 \mu \mathrm{m} / \mu \mathrm{m}$. Bending angle $180^{\circ}$. c Stretching conductivity of EGaln-rich layer on SEBS. EGaln layer: $10 \mu \mathrm{m}$. The inset gives optical observation before (Left) and after (Middle \& Right) stretching. d Electromagnetic shielding efficiency (EM SE) of EGaln-rich layer after sintering. EGaln/CNFs layer thickness: 10/10 $\mu \mathrm{m} / \mu \mathrm{m}$. e Comparison of SE/t as function of thickness $t .1: \mathrm{Ti}_{3} \mathrm{C}_{2} \mathrm{~T}_{x} /$ cellulose $^{26} ; 2$ : Graphene/PDMS $32 ; 3$ : Reduced graphene oxide $(\mathrm{rGO})^{37} ;$ 4: Carbon black/rubber28; 5: Carbon nanowires/ graphene/PDMS 34; 6: Carbon nanotube (CNT)/acrylonitrile-butadiene-styrene ${ }^{29}$; 7: Steel/PP33; 8: rGO/poly(3,4-ethylenedioxythiophene) ${ }^{31}$; 9: rGO/ polyetherimide ${ }^{30}$; 10: Carbon/phthalonitrile ${ }^{36} ; 11$ : Ni/polyethersulfone ${ }^{35} ; 12$ : Carbon nanofibers ${ }^{27}$. $\mathbf{f}$ Biological degradation of free-standing film in soil extract for 0 and 15 days

stretching up to $100 \%$ (Supplementary Fig. 22). This suggested that the remaining high-aspect-ratio CNFs enabled stretchability of the conductive EGaIn-rich layer. Their polar groups also ensured the EGaIn affinity to the substrate.

Besides serving for flexible electronics, this conductive layer also exhibited an electromagnetic shielding property with a comprehensive shielding efficiency $(\mathrm{SE}) \geq 12 \mathrm{~dB}$ (for the layer thickness of only $\sim 20 \mu \mathrm{m}$ ) in the X-band frequency range of 8.2-12.4 GHz (Fig. 2d). In spite of fluctuations across the tested frequency range, its overall performance is comparable or superior to many reported materials with higher thicknesses (Fig. 2e), thus being ideal for electromagnetic shielding applications ${ }^{26-37}$. Furthermore, the bilayer film and substrate coating could fully degrade in natural soil extract within 15 days (Fig. $2 \mathrm{f}$ and Supplementary Fig. 23), owing to biological and/or chemical degradation of CNFs and EGaIn. Thus this unique type of materials would offer an alternative for degradable and flexible electronics.

Responsive actuating behaviour. The prepared free-standing films possessed an intrinsic Janus feature, in which both the bottom and top layers showed distinct responsibility to humidity and electricity. For example, owing to different hydration abilities of CNFs and EGaIn, the Janus film delivered an unique actuating behaviour under cyclically exposing to different humidities (e.g., RH 40 and $100 \%$ ), alike to reversible movement in pine cones (Supplementary Fig. 24) ${ }^{38}$. The actuating curvature maximised at the thickness ratio $\sim 0.9$ of the EGaIn-rich and CNFs-rich layers (see detailed calculation in Supporting Information and
Supplementary Fig. 24c). Moreover, the conductive EGaIn layer had a strong electro-thermal effect, being capable of heating under a low voltage (e.g., $\geq 25^{\circ} \mathrm{C}$ within $3 \mathrm{~s}$ under $2.5 \mathrm{~V}$ in Fig. $3 \mathrm{a}$ ). This Joule-heating effect would also enable an electrical actuating behaviour: A U-shaped bilayer film $\left(24 \times 2 \mathrm{~mm}^{2}\right.$ with a middle space of $0.2 \mathrm{~mm}$ wide; $9 / 10 \mu \mathrm{m} / \mu \mathrm{m}$ for EGaIn/CNFs thickness) bended up to $360^{\circ}$ within $3 \mathrm{~s}$ under a constant bias of $2.0 \mathrm{~V}$ ( $\mathrm{RH} \sim 70 \%)$, whose bending angle was tuneable by the applying voltage (Fig. 3b, Supplementary Fig. 25 and Supplementary Movie 2). When switching off the voltage, the bending angle recovered in several seconds (Fig. 3c). In analogue, this actuating behaviour was driven by cyclic dehydration and hydration of the CNFs-rich layer, in which the Joule-heating effect would dehydrate the CNFs-rich layer (Fig. 3d). This actuating behaviour showed a large bending displacement in analogue to natural flowers as well as rapidity nearly comparable to predatory motion of cabrites (Fig. 3e and Supplementary Movie 3). This actuating speed is also superior to many reported soft actuators, in the form of bilayer-structures like shape-memory and ionic polymeric composites (Fig. 3f) ${ }^{39-51}$.

There might exist small EGaIn droplets (with the diameter $<200$ $\mathrm{nm}$ ) within the CNFs-rich layer (Fig. 4a), whose photo-thermal effect had been reported in the literature ${ }^{52}$. This photo-thermal effect could heat the Janus film when exposing to photo radiation (Fig. 4b and Supplementary Fig. 26), and enable an actuating behaviour responsive to photo. Under a near-infrared light radiation (Wavelength of $808 \mathrm{~nm}$ and light density of $0.8 \mathrm{~W} \mathrm{~cm}$ ), a Janus film $\left(20 \times 1 \mathrm{~mm}^{2}\right.$; thickness: $9 / 10 \mu \mathrm{m} / \mu \mathrm{m}$ for EGaIn/CNFs $)$ bended up to $90^{\circ}$ within $2 \mathrm{~s}$ and recover back within $<2.5 \mathrm{~s}$ (Fig. $4 \mathrm{c}$ ). This 

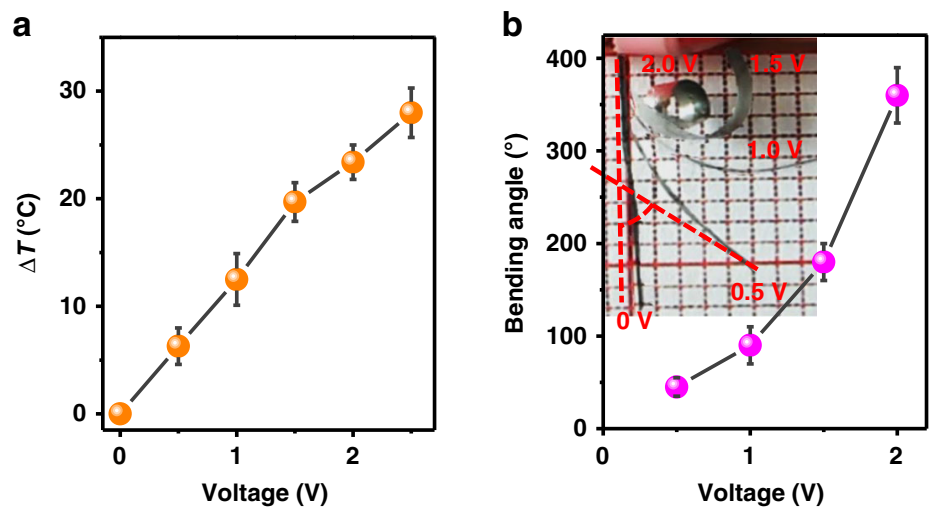

d

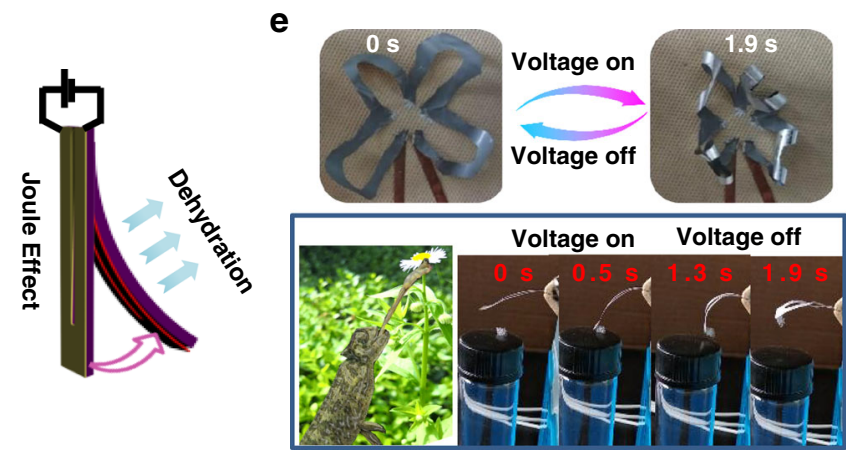

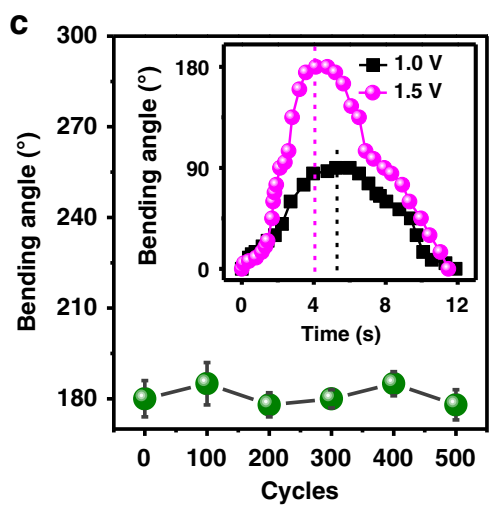

f

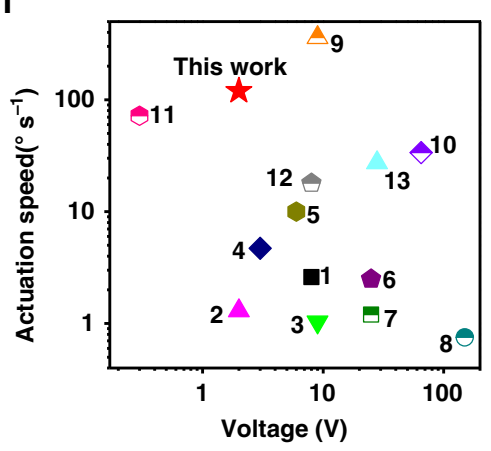

Fig. 3 Electrically-driven actuating behaviour of Janus film. a Joule-heating effect at different voltages. $25^{\circ} \mathrm{C}$; Joule-heating effect ( $\Delta T$ : elevated temperature from $25^{\circ} \mathrm{C}$ ) at different voltages. Rectangular film of $24 \times 2 \mathrm{~mm}^{2}$; EGaln/CNFs layer thickness: $9 / 10 \mu \mathrm{m} / \mu \mathrm{m}$. $\mathbf{b}$ Bending angles at different voltages. $\mathrm{RH}: 70 \%$. c Repeatable bending under at voltage of $1.5 \mathrm{~V}$. The inset shows temporal variation of bending angle at constant voltage of 1.0 and $1.5 \mathrm{~V}$. d Actuating mechanism: Dehydrating CNFs-rich layer by Joule-effect of EGaln-rich layer. e Actuating behaviour in analogue to flower blossom and

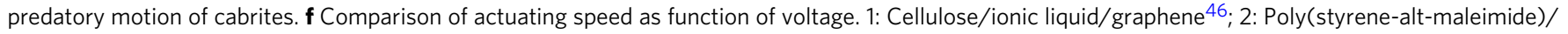
poly(vinylidene fluoride)40; 3: Poly(3,4-ethylenedioxythiophene)/ poly(styrene sulfonate)/ ionic liquids ${ }^{47}$; 4: Chitosan/CNT39; 5: rGO/epoxy42; 6:

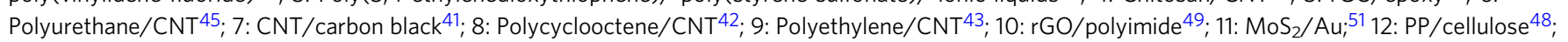
13: Graphene/Ag/polyimide 50

bending-and-recovering behaviour could repeat for hundreds of times without showing obvious bending decay. Moreover, a larger bending displacement and larger velocity were produced by higher power density of NIR radiation (Fig. 4d). Besides these, this photo-thermal effect can be used to twist the bilayer film in analogue to the bean pod (Supplementary Movie 4), and drive an origami (Fig. 4e and Supplementary Movie 5). It could drive floating displacement of the Janus film on water (Fig. $4 \mathrm{f}$ and Supplementary Movie 6), because of locally heating surrounding water ${ }^{38}$.

\section{Discussion}

With the presence of biological NFs, EGaIn droplets underwent a precipitation process together with an evaporation-induced sintering process, offering an insulating NFs-rich layer over a conductive EGaIn-rich layer (with a conductivity up to $\sim 10^{6} \mathrm{~S} \mathrm{~m}^{-1}$ with the layer thickness of $>5 \mu \mathrm{m}$ in Fig. $5 \mathrm{a}$ ). This precipitation separation process seemed to be essential for sintering. For instance, no electric conductivity was achieved when rapidly removing the solvent through filtration and rapid drying (Supplementary Fig. 13d and 16b). The presence of CNFs was also essential for this evaporation-induced sintering process. In the suspension, the CNFs concentration as low as $0.05 \mathrm{wt} \%$ could assist to sinter EGaIn droplets to achieve a metallic conductivity up to $2 \times 10^{5} \mathrm{~S} \mathrm{~m}^{-1}$ (Fig. 5b \& Supplementary Fig. 27). And high CNFs concentrations below $0.3 \mathrm{wt} \%$ did not influence electric conductivity of the final EGaIn layer, probably due to their separation from the sedimental EGaIn droplets. In addition, no evaporation-induced sintering occurred when removing free CNFs by centrifuging the EGaIn droplets (Supplementary Fig. 28).

During the sintering, CNFs unambiguously produced a local pressure large enough to rupture the encapsulating shells of EGaIn droplets, in analogue to mechanical sintering. It would produce a sharp conductivity increase (Supplementary Fig. 18). When consolidating colloidal particles, evaporation is known to give a strong capillary force as ${ }^{53}$ :

$$
F_{\text {capillary }}=2 \pi \gamma \alpha \sin \varphi \sin (\varphi+\theta)+\pi \alpha^{2} \sin ^{2} \varphi \Delta P
$$

where the first and second terms are the force arising from the surface tension and the Laplace pressure, respectively; $\varphi$ is the half-filling angle; $\gamma$ is the surface tension of the liquid; $\theta$ is the liquid-solid contact angle; $\alpha$ is the sphere radius; $\Delta P$ is the pressure difference cross the liquid surfaces, and identified as the Young-Laplace equation:

$$
\Delta P=\frac{2 \gamma \cos \theta}{r_{p}}
$$

where $r_{\mathrm{p}}$ is the capillary curvature radius. The capillary forces could also be represented as ${ }^{21}$ :

$$
F_{\text {capillary }}=2 \pi \gamma \alpha \sin \varphi \sin (\varphi+\theta)+2 \pi \gamma \alpha \cos \theta /[1+H / 2 d]
$$

where $H$ is the shortest distance between the spheres; $d$ is the immersion length of the sphere and calculated by $d=(H / 2) \times\left[-1+\sqrt{1+2 V /\left(\pi \alpha H^{2}\right)}\right]$, where $V$ is the volume of liquid bridge. Colloidal agglomeration and coalescence driven by 
a

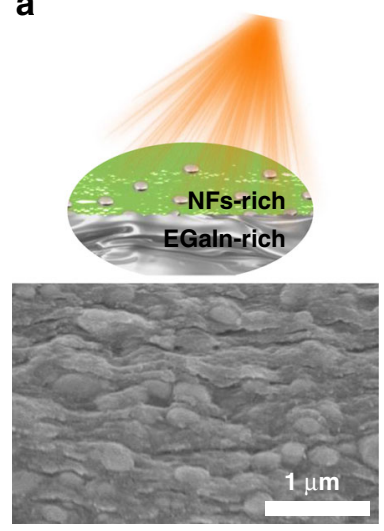

d

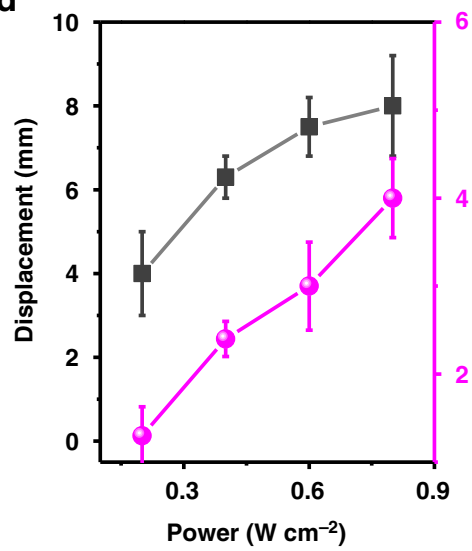

b

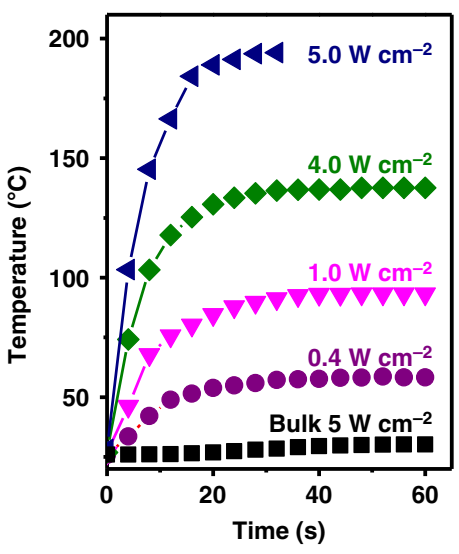

e
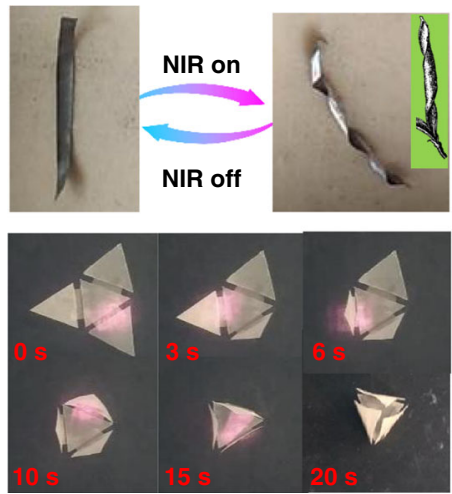

C
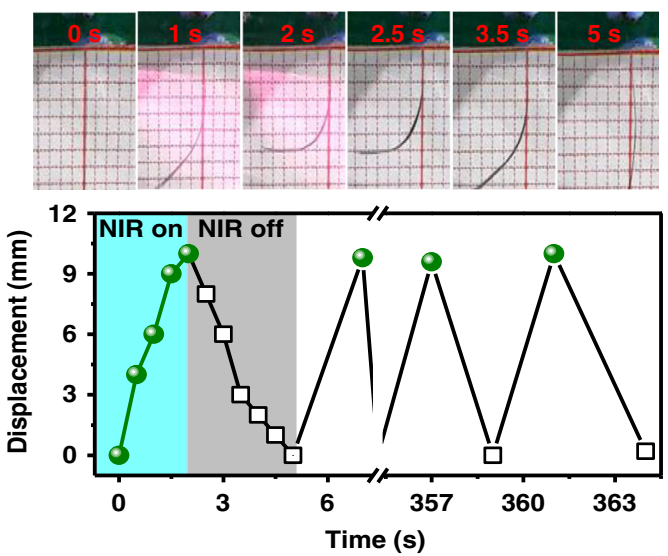

f

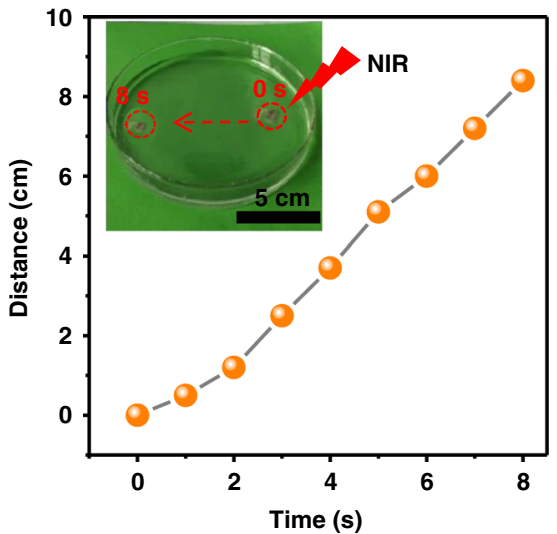

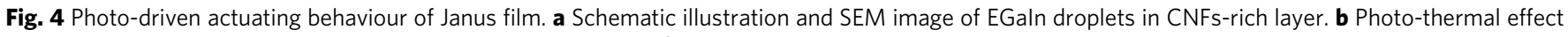
at different NIR power densities. Rectangular film of $20 \times 10 \mathrm{~mm}^{2}$; EGaln/CNFs layer thickness: $9 / 10 \mu \mathrm{m} / \mu \mathrm{m}$. NIR wavelength: $808 \mathrm{~nm}$. Bulk EGaln was used as the control. c Visual observation (Top) and repeatability (Bottom) of actuating behaviour under NIR irradiation. Power density of NIR: 0.8 W $\mathrm{cm}$-2. d Displacement and actuating speed at different NIR power densities. e Photo-driven self-twisting (Top) and origami (Bottom). The inset (top right) shows twisted Vigna Radiata pods. f Photo-driving floating displacement in water. Energy density of radiation: $0.8 \mathrm{~W} \mathrm{~cm}^{-2}$

capillary forces widely exist in nature (e.g., biological adhesion) and colloidal technologies (e.g., inkjet printing, and self-assembly of colloidal particles) ${ }^{54,55}$. During evaporation of solvents, meniscus-shaped capillary bridges would form among the colloids and yield an attraction force ${ }^{20,55}$.

Firstly, the presence of CNFs might promote $F_{\text {capillary }}$ between the EGaIn droplets by decreasing their sizes and contact angles. The smaller droplet size favoured larger $\Delta P$ due to the larger capillary curvature (Fig. $5 \mathrm{c}$ ). The attached CNFs favoured a smaller contact angle and hereby larger $\Delta P$. The increasing $\Delta P$ would contribute dominantly to $F_{\text {capillary }}$ between the EGaIn droplets. For example, by assuming $\alpha=60 \mathrm{~nm}, V=10^{3} \mathrm{~nm}^{3}$, $H=5 \mathrm{~nm}$ and $\theta=5^{\circ 56}, F_{\text {capillary }}$ would produce a local pressure up to $\sim 13.7 \mathrm{MPa}$ on EGaIn droplets according to Equation 3 . While only a pressure of $\sim 0.9 \mathrm{MPa}$ was obtained in absence of CNFs (detailed in Supporting Information See details in Supplementary Note 1).

Secondly, the presence of CNFs might also split the liquid bridges between the droplets. Spitting liquid bridges into small ones might lead to an increase of the capillary forces (Fig. $5 \mathrm{~d})^{57}$. In many biological wet adhesive systems, the adhesion forces is not only a function of contact angle, but also a nonlinear function of the number and size of liquid bridges. Multiple liquid bridges could generate the highest capillary forces without increasing the total contact area, which may exceed van der Waals forces by several orders of magnitude. At the same time, evaporation also generated capillary forces among the CNFs attaching on EGaIn droplets, which would produce a local pulling tension on the order of magnitude over $10 \mathrm{MPa}^{20}$. Due to the large elastic modulus of CNFs up to $10^{2} \mathrm{GPa}$, this pulling tension could transmit along the CNFs and exert on the droplet shells.

Without the presence of CNFs, capillary forces among the EGaIn droplets were not sufficient to rupture their oxide shells. Thus these droplets deposited on the substrate as an insulating porous layer after completely drying (Fig. 5e and Supplementary Fig. 13). When further casting a suspension (e.g., $\phi_{\mathrm{CNF}}=0.2 \mathrm{wt} \%$ ) of CNFs on the top, a portion of CNFs would infiltrate into the gaps among the EGaIn droplets, and sinter them during the following evaporation (Fig. 5e). Notably, there possibly existed a thin layer of EGaIn droplets between the bottom conductive layer and the top CNFs-rich layer, in which excess CNFs might form densely packing shells and prevent the droplet coalescence.

In summary, biological NFs downsized and enabled evaporation-induced sintering of LM droplets under ambient conditions into conductive free-standing functional films and coatings on substrates as diverse as rigid, soft, biological and in microfluidic channels, in sharp contrast to laser sintering on heatresistant substrates and mechanical sintering on rigid substrates. The contribution of biological NFs was threefold: (a) Attaching on the surfaces of EGaIn droplets for lower droplet size (down to $50 \mathrm{~nm}$ in diameter) and higher colloidal stability in the suspension; (b) During evaporation-induced sintering, biological NFs as 
a

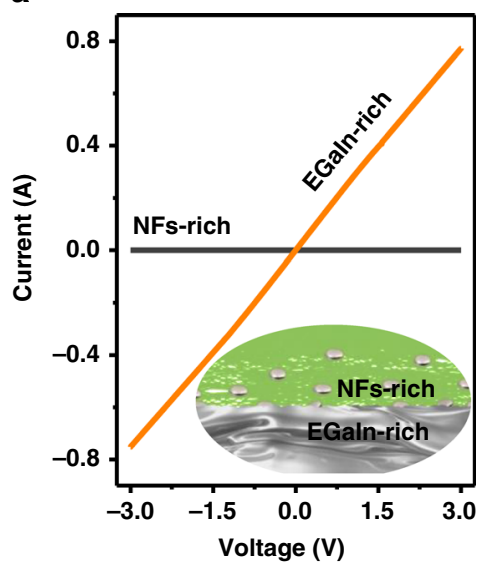

d

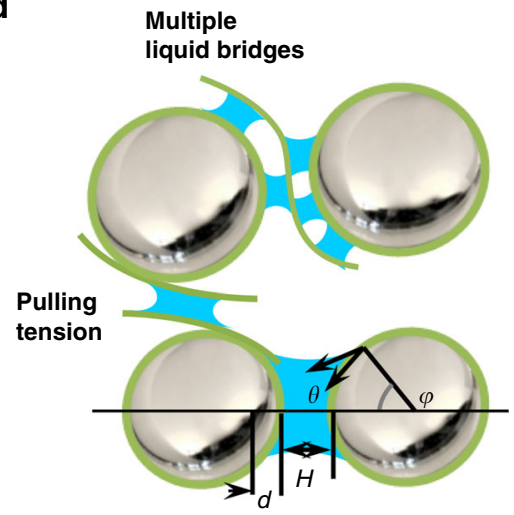

b

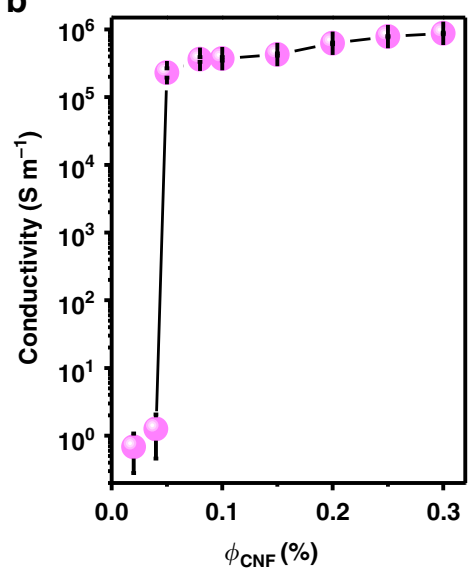

C

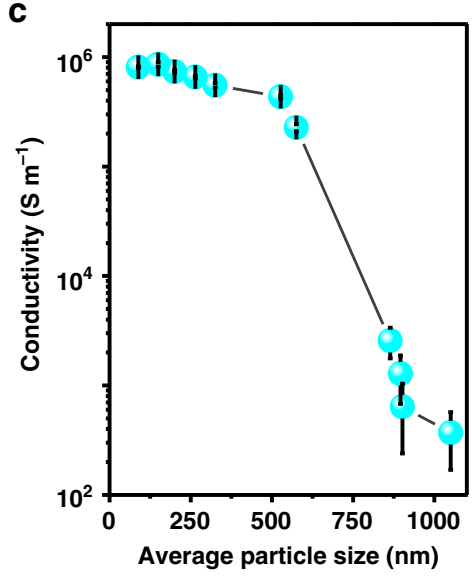

e

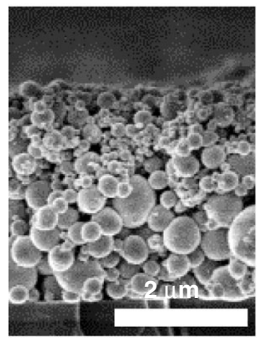

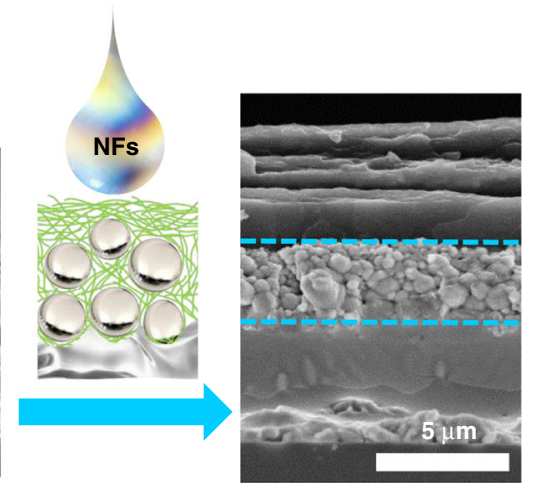

Higher capillary force

Fig. 5 Evaporation-induced sintering mechanism of EGaln droplets with biological NFs. a $1-V$ curves of CNFs-rich and EGaln-rich sides of free-standing films. EGaln/CNFs layer thickness: $9 / 10 \mu \mathrm{m} / \mu \mathrm{m}$. b Effect of CNFs concentration on evaporation-induced sintering evaluated by conductivity of EGaln-rich side. Average EGaln droplet size: $120 \mathrm{~nm}$. c Size effect of EGaln droplets on evaporation-induced sintering evaluated by conductivity of EGaln-rich side. d Schematic illustration of sintering mechanism: Capillary force promoted by biological NFs; Local pulling tension induced by biological NFs; Splitting into multiple liquid bridges. e Depositing NFs suspension on layer of EGaln droplets produced without NFs for evaporation-induced sintering

low as $0.05 \mathrm{wt} \%$ might promote capillary forces and split liquid bridges among EGaIn droplets, and as well create high pull tension on the shells of EGaIn droplets. (c) Biological NFs had sufficient polar groups (i.e., carboxyl and hydroxyl), which also ensured adhesion and stretchability of EGaIn layers on diverse substrates by printing, casting and depositing, and thus could serve as structural matrix of EGaIn droplets for free-standing, humidity responsivity, biocompatibility and biodegradation in electronic applications. With the coexistence of NFs-rich and EGaIn-rich layers, both the resultant coating and free-standing films showed flexible conductivity (e.g., with conductivity up to $10^{5} \mathrm{~S} \mathrm{~m}^{-1}$ at $200 \%$ strain), mirror-like reflectivity and electromagnetic-shield, more importantly, whose actuating behaviours could response to humidity, photo and voltage. Thus this evaporation-induced approach not only extends fundamental knowledge of LM droplets and their suspensions, but also starts a new scenario of producing flexible coating and free-standing composites with flexibility, conductivity, sustainability and degradability, and applicable in microcircuits, sensing, wearable devices and soft robotics.

\section{Methods}

Materials. EGaIn was provided by Shenyang Jiabei Trading Co., Ltd. (China). Needle bleached kraft pulp was bought from Hangzhou Wohua Filter Paper Co. Ltd. TEMPO was supplied by Sigma-Aldrich. Sodium hypochlorite solution (12 wt\%) and $\mathrm{NaBr}$ were purchased from Aladdin Industrial Co. Ltd. Ultrapure water (resistivity $18.2 \mathrm{M} \Omega \mathrm{cm}^{-1}$ ) was used to prepare all aqueous solutions.
Detailed preparation of aqueous dispersions of biological NFs (including cellulose, fibroin and amyloid) was shown in Supplementary Methods.

Preparation of EGaln droplets. Typically, bulk EGaIn (150 mg) was added to an aqueous solution $(15 \mathrm{~mL}$ ) of NFs. The mixture underwent sonication (BILON92-II; power of $300 \mathrm{~W}$ with $80 \%$ amplitude) in ice-bath water with different NFs concentrations and time periods. Size-grading was achieved by centrifuging. And free NFs in the dispersion were removed by centrifugation at 10,000 rpm.

Evaporation-induced sintering. The as-dispersed EGaIn droplets $\left(\phi_{\mathrm{CNF}} 0.04-0.3\right.$ wt $\%$, EGaIn concentration $10 \mathrm{mg} \mathrm{mL}^{-1}$, and average droplet size $100-850 \mathrm{~nm}$ ) were deposited on the substrate through drop-casting, direct hand-writing and spraycoating, following by drying at ambient conditions $\left(\sim 0.1 \mathrm{MPa}, 25 \pm 3^{\circ} \mathrm{C}, \mathrm{RH} 40 \pm\right.$ $5 \%$ ) at least for one day for completely drying before further test. Bulk conductivity of the dried trace was measured to evaluate the sintering status of the deposited EGaIn droplets. The thickness and resistance of the dried trace were measured by SEM and digital multimeter (MS8265), respectively. For sintering on soft substrates, vacuum drying $(-70 \mathrm{kPa})$ was applied. When the layer thickness of CNFs was sufficiently large, the coating on glass substrates could peeled off as freestanding films.

Actuating test. For voltage-responsive actuator, a Janus film was cut into a $\mathrm{U}$ shape strip with a rectangle of $24 \times 2 \mathrm{~mm}^{2}$ and a middle space of $0.2 \mathrm{~mm}$ wide. Copper wires were connected to two actuator ends with Ag paste. Bending angles were recorded at the voltage of $0.5-2.0 \mathrm{~V}$ at RH $70 \%$. Cyclic actuating performance was carried out at $2.0 \mathrm{~V}$. For photo-responsive actuator, a Janus film $\left(20 \times 1 \mathrm{~mm}^{2}\right)$ was equilibrated at $25^{\circ} \mathrm{C}$ and $\mathrm{RH} \sim 70 \%$ for $24 \mathrm{~h}$. A NIR laser with tunable power (wavelength of $808 \mathrm{~nm}$, maximum output power of $1.0 \mathrm{~W}$, Xi'an Minghui Optoelectronic Technology Co., Ltd.) was used as the NIR source. 
Stretchable conductivity test. A sintered coating (with $9 / 20 \mu \mathrm{m} / \mu \mathrm{m}$ in thickness of CNFs and EGaIn) was prepared on a strip of SEBS $\left(20 \times 5 \times 0.5 \mathrm{~mm}^{3}\right)$. The stretching test was performed on a mechanical tester at a speed of $2 \mathrm{~mm} \mathrm{~min}^{-1}$.

Electromagnetic shielding test. The performance was measured on an Agilent N5227A vector network analyser using an APC-7 connector as the coaxial test cell in the X-band frequency range of $8.2-12.4 \mathrm{GHz}$. The background from the substrate was deducted according to a simple addition rule.

Characterisation. Field emission scanning electron microscopy (FESEM, Hitachi S-4800, Japan) with X-ray energy dispersive spectrometry (EDS) was used to characterise microstructure and elemental mapping at an acceleration voltage of $10 \mathrm{kV}$. TEM (Hitachi H-7650) measurements were performed at a voltage of $100 \mathrm{kV}$. Tensile experiments were performed on Electromechanical Universal Testing Machine (CMT6503, MTS Industrial Systems Co., Ltd. China). Photo reflection spectra were recorded in the visible light region $(400-720 \mathrm{~nm})$ with a Hitachi U-4100 spectrophotometer (Japan).

\section{Data availability}

Data supporting the findings of this work are available within the paper and its Supplementary Information files and from the corresponding authors upon reasonable request.

Received: 19 February 2019 Accepted: 25 June 2019

Published online: 05 August 2019

\section{References}

1. Wang, S. et al. Skin electronics from scalable fabrication of an intrinsically stretchable transistor array. Nature 555, 83-88 (2018).

2. Zhu, B., Gong, S. \& Cheng, W. Softening gold for elastronics. Chem. Soc. Rev. 48, 1668-1711 (2019).

3. Choi, S., Lee, H., Ghaffari, R., Hyeon, T. \& Kim, D. H. Recent advances in flexible and stretchable bio-electronic devices integrated with nanomaterials. Adv. Mater. 28, 4203-4218 (2016).

4. Li, C., Adamcik, J. \& Mezzenga, R. Biodegradable nanocomposites of amyloid fibrils and graphene with shape-memory and enzyme-sensing properties. Nat. Nanotechnol. 7, 421-427 (2012).

5. Wang, C., Wang, C., Huang, Z. \& Xu, S. Materials and structures toward soft electronics. Adv. Mater. 30, e1801368 (2018).

6. Jang, H. et al. Graphene-based flexible and stretchable electronics. Adv. Mater. 28, 4184-4202 (2016).

7. Kim, D. H. \& Rogers, J. A. Stretchable electronics: Materials strategies and devices. Adv. Mater. 20, 4887-4892 (2010).

8. Boley, J. W., White, E. L. \& Kramer, R. K. Mechanically sintered galliumindium nanoparticles. Adv. Mater. 27, 2355-2360 (2015).

9. Yoonseob, K. et al. Stretchable nanoparticle conductors with self-organized conductive pathways. Nature 500, 59-63 (2013).

10. Li, Y. et al. A review of the electrical and mechanical properties of carbon nanofiller-reinforced polymer composites. J. Mater. Sci. 54, 1036-1076 (2018).

11. $\mathrm{Li}, \mathrm{X}$. et al. Liquid metal droplets wrapped with polysaccharide microgel as biocompatible aqueous ink for flexible conductive devices. Adv. Funct. Mater. 28, 1804197 (2018).

12. Kazem, N., Hellebrekers, T. \& Majidi, C. Soft multifunctional composites and emulsions with liquid metals. Adv. Mater. 29, 1605985 (2017).

13. Hohman, J. N. et al. Directing substrate morphology via self-assembly: Ligand-mediated scission of gallium-indium microspheres to the nanoscale. Nano Lett. 11, 5104-5110 (2011).

14. Khoshmanesh, K. et al. Liquid metal enabled microfluidics. Lab Chip 17, 974-993 (2017).

15. Zhang, B. et al. Self-powered acceleration sensor based on liquid metal triboelectric nanogenerator for vibration monitoring. ACS Nano 11, 7440-7446 (2017).

16. Wang, J. et al. Printable superelastic conductors with extreme stretchability and robust cycling endurance enabled by liquid-metal particles. Adv. Mater 30, 1706157 (2018)

17. Lin, Y. et al. Handwritten, soft circuit boards and antennas using liquid metal nanoparticles. Small 11, 6397-6403 (2015).

18. Liu, S. et al. Laser sintering of liquid metal nanoparticles for scalable manufacturing of soft and flexible electronics. ACS Appl. Mater. Interfaces 10, 28232-28241 (2018)

19. Deng, B. \& Cheng, G. J. Pulsed laser modulated shock transition from liquid metal nanoparticles to mechanically and thermally robust solid-liquid patterns. Adv. Mater., 31, 1807811 (2019).
20. Liu, Y. et al. Capillary-force-induced cold welding in silver-nanowire-based flexible transparent electrodes. Nano Lett. 17, 1090-1096 (2017).

21. Rabinovich, Y. I., Esayanur, M. S. \& Moudgil, B. M. Capillary forces between two spheres with a fixed volume liquid bridge: Theory and experiment. Langmuir 21, 10992-10997 (2005).

22. Ling, S. et al. Biopolymer nanofibrils: Structure, modeling, preparation, and applications. Prog. Polym. Sci. 85, 1-56 (2018).

23. $\mathrm{Wu}, \mathrm{X}$. et al. Amyloid-graphene oxide as immobilization platform of $\mathrm{Au}$ nanocatalysts and enzymes for improved glucose-sensing activity. J. Colloid Inter. Sci. 490, 336-342 (2017).

24. Lv, L. et al. Biomimetic hybridization of Kevlar into silk fibroin: Nanofibrous strategy for improved mechanic properties of flexible composites and filtration membranes. ACS Nano 11, 8178-8184 (2017).

25. Zhu, L. et al. Shapeable fibrous aerogels of metal-organic-frameworks templated with nanocellulose for rapid and large-capacity adsorption. ACS Nano 12, 4462-4468 (2018).

26. Cao, W. T. et al. Binary strengthening and toughening of MXene/cellulose nanofiber composite paper with nacre-inspired structure and superior electromagnetic interference shielding properties. ACS Nano 12, 4583-4593 (2018).

27. Hong, X. \& Chung, D. D. L. Carbon nanofiber mats for electromagnetic interference shielding. Carbon 111, 529-537 (2017).

28. Ghosh, P. \& Chakrabarti, A. Conducting carbon black filled EPDM vulcanizates: Assessment of dependence of physical and mechanical properties and conducting character on variation of filler loading. Eur. Polym. J. 36, 1043-1054 (2000).

29. Al-Saleh, M. H., Saadeh, W. H. \& Sundararaj, U. EMI shielding effectiveness of carbon based nanostructured polymeric materials: A comparative study. Carbon 60, 146-156 (2013)

30. Ling, J. et al. Facile preparation of lightweight microcellular polyetherimide/ graphene composite foams for electromagnetic interference shielding. ACS Appl. Mater. Interfaces 5, 2677-2684 (2013).

31. Agnihotri, N., Chakrabarti, K. \& De, A. Highly efficient electromagnetic interference shielding using graphite nanoplatelet/poly $(3,4$ ethylenedioxythiophene)-poly(styrenesulfonate) composites with enhanced thermal conductivity. RSC Adv. 5, 43765-43771 (2015).

32. Chen, Z., Xu, C., Ma, C. L., Ren, W. \& Cheng, H. W. Lightweight and flexible graphene foam composites for high-performance electromagnetic interference shielding. Adv. Mater. 25, 1296-1300 (2013).

33. Ameli, A., Nofar, M., Wang, S. \& Park, C. B. Lightweight polypropylene/ stainless-steel fiber composite foams with low percolation for efficient electromagnetic interference shielding. ACS Appl. Mater. Interfaces 6, 11091-11100 (2014)

34. Luo, K. et al. Macroscopic bioinspired graphene sponge modified with in-situ grown carbon nanowires and its electromagnetic properties. Carbon 111, 94-102 (2017).

35. Song, W. L. et al. Magnetic and conductive graphene papers toward thin layers of effective electromagnetic shielding. J. Mater. Chem. A 3, 2097-2107 (2015).

36. Zhang, L. et al. Phthalonitrile-based carbon foam with high specific mechanical strength and superior electromagnetic interference shielding performance. ACS Appl. Mater. Interfaces 8, 7422-7430 (2016).

37. Yan, D. X. et al. Structured reduced graphene oxide/polymer composites for ultra-efficient electromagnetic interference shielding. Adv. Funct. Mater. 25, 559-566 (2015)

38. Zong, L., Li, M. \& Li, C. Bioinspired coupling of inorganic layered nanomaterials with marine polysaccharides for efficient aqueous exfoliation and smart actuating hybrids. Adv. Mater. 29, 1604691 (2017).

39. Lu, L. \& Chen, W. Biocompatible composite actuator: A supramolecular structure consisting of the biopolymer chitosan, carbon nanotubes, and an ionic liquid. Adv. Mater. 22, 3745-3748 (2010).

40. Lu, J., Kim, S.-G., Lee, S. \& Oh, I.-K. A biomimetic actuator based on an ionic networking membrane of poly(styrene-alt-maleimide)-incorporated poly (vinylidene fluoride). Adv. Funct. Mater. 18, 1290-1298 (2008).

41. Yu, K., Zhang, Z., Liu, Y. \& Leng, J. Carbon nanotube chains in a shape memory polymer/carbon black composite: To significantly reduce the electrical resistivity. Appl. Phys. Lett. 98, 074102 (2011).

42. Wang, Z. et al. Dually actuated triple shape memory polymers of cross-linked polycyclooctene-carbon nanotube/polyethylene nanocomposites. ACS Appl. Mater. Interfaces 6, 20051-20059 (2014).

43. Li, L. et al. Dual-mechanism and multimotion soft actuators based on commercial plastic film. ACS Appl. Mater. Interfaces 17, 15122-15128 (2018).

44. Wang, W., Liu, D., Liu, Y., Leng, J. \& Bhattacharyya, D. Electrical actuation properties of reduced graphene oxide paper/epoxy-based shape memory composites. Compos. Sci. Technol. 106, 20-24 (2015).

45. Sahoo, N. G., Jung, Y. C. \& Cho, J. W. Electroactive shape memory effect of polyurethane composites filled with carbon nanotubes and conducting polymer. Mater. Manuf. Process. 22, 419-423 (2007). 
46. Kim, S.-S., Jeon, J.-H., Kim, H.-I., Kee, C. D. \& Oh, I.-K. High-fidelity bioelectronic muscular actuator based on graphene-mediated and TEMPO-oxidized bacterial cellulose. Adv. Funct. Mater. 25, 3560-3570 (2015).

47. Wang, F. et al. High-fidelity bioelectronic muscular actuator based on porous carboxylate bacterial cellulose membrane. Sens. Actuators B-Chem. 250, 402-411 (2017).

48. Amjadi, M. \& Sitti, M. High-performance multiresponsive paper actuators. ACS Nano 10, 10202-10210 (2016)

49. Zhang, T. Y. et al. A large-strain, fast-response, and easy-to-manufacture electrothermal actuator based on laser-reduced graphene oxide. Appl. Phys. Lett. 111, 121901 (2017).

50. Wang, Q. et al. Low-voltage, large-strain soft electrothermal actuators based on laser-reduced graphene oxide/Ag particle composites. Appl. Phys. Lett. 112, 133902 (2018).

51. Acerce, M., Akdogan, E. K. \& Chhowalla, M. Metallic molybdenum disulfide nanosheet-based electrochemical actuators. Nature 549, 370-373 (2017).

52. Chechetka, S. A. et al. Light-driven liquid metal nanotransformers for biomedical theranostics. Nat. Commun. 8, 15432 (2017).

53. Zhou, Z., Li, Q. \& Zhao, X. S. Evolution of interparticle capillary forces during drying of colloidal crystals. Langmuir 22, 3692-3697 (2006).

54. Ramos, J. R. D., Travasso, R. \& Carvalho, J. Capillary network formation from dispersed endothelial cells: Influence of cell traction, cell adhesion, and extracellular matrix rigidity. Phys. Rev. E 97, 012408 (2018).

55. Sang-Wook, L., Seung Chul, P., Yongjun, L., Byoungho, L. \& Sin-Doo, L. Polymorphic meniscus convergence for construction of quasi-periodic assemblies and networks of colloidal nanoparticles. Adv. Mater. 22, 4172-4175 (2010).

56. Jia, Y. et al. Surfactant-free emulsions stabilized by tempo-oxidized bacterial cellulose. Carbohydr. Polym. 151, 907-915 (2016).

57. De Souza, E. J., Brinkmann, M., Mohrdieck, C. \& Arzt, E. Enhancement of capillary forces by multiple liquid bridges. Langmuir 24, 8813-8820 (2008).

\section{Acknowledgements}

National Natural Science Foundation of China (Nos. 21474125 and 51608509), Chinese "1000 Youth Talent Program", Shandong "Taishan Youth Scholar Program”, Shandong Provincial Natural Science Foundation (Nos. JQ201609 and ZR2016EEB25), “135” Projects Fund of CAS QIBEBT Director Innovation Foundation and Shandong Collaborative Innovation Centre for Marine Biomass Fiber Materials and Textiles are kindly acknowledged for financial support. The authors also thank Dr. Anle Ge in Qingdao
Institute of Bioenergy and Bioprocess Technology, Chinese Academy of Sciences for the contribution to preparation of microfluidic channels and Prof. Shu Lin in Harbin Institute of Technology for the contribution to analysis of electromagnetic interference shielding results.

\section{Author contributions}

X.L., M. L, J.X., J.Y. and Z.Y. performed the experiments. C.L. and M.L performed the analysis and wrote the paper. C.L. directed this study.

\section{Additional information}

Supplementary Information accompanies this paper at https://doi.org/10.1038/s41467019-11466-5.

Competing interests: The authors declare no competing interests.

Reprints and permission information is available online at http://npg.nature.com/ reprintsandpermissions/

Peer review information: Nature Communications thanks Khashayar Khoshmanesh, Alexander Mukasyan and other anonymous reviewer(s) for their contribution to the peer review of this work. Peer reviewer reports are available.

Publisher's note: Springer Nature remains neutral with regard to jurisdictional claims in published maps and institutional affiliations.

(c) (i) Open Access This article is licensed under a Creative Commons Attribution 4.0 International License, which permits use, sharing, adaptation, distribution and reproduction in any medium or format, as long as you give appropriate credit to the original author(s) and the source, provide a link to the Creative Commons license, and indicate if changes were made. The images or other third party material in this article are included in the article's Creative Commons license, unless indicated otherwise in a credit line to the material. If material is not included in the article's Creative Commons license and your intended use is not permitted by statutory regulation or exceeds the permitted use, you will need to obtain permission directly from the copyright holder. To view a copy of this license, visit http://creativecommons.org/ licenses/by/4.0/.

(C) The Author(s) 2019 